\title{
Exploring Physiotherapists' Use of Clinical Practice Guidelines, Screening, and Stratification Tools for People with Low Back Pain in New Zealand
}

\author{
Julia Hill $P h D$ \\ Department of Physiotherapy; Active Living and Rehabilitation: Aotearoa New Zealand, School of Clinical Sciences, Auckland \\ University of Technology, Auckland, New Zealand
}

John Bedford BHSC

Department of Physiotherapy, School of Clinical Sciences, Auckland University of Technology, Auckland, New Zealand

David Houston BHSC

Department of Physiotherapy, School of Clinical Sciences, Auckland University of Technology, Auckland, New Zealand

\section{Duncan A. Reid DHSC}

Department of Physiotherapy; Active Living and Rehabilitation: Aotearoa New Zealand, School of Clinical Sciences, Auckland University of Technology, Auckland, New Zealand

G. David Baxter TC, BSC(Hons), DPhil, MBA, FCSP

Centre for Health, Activity and Rehabilitation Research, School of Physiotherapy, University of Otago, Dunedin, New Zealand

\section{Richard Ellis $P h D$}

Department of Physiotherapy; Active Living and Rehabilitation: Aotearoa New Zealand, School of Clinical Sciences, Auckland University of Technology, Auckland, New Zealand

\section{ABSTRACT}

Low back pain (LBP) is a leading cause of disability in New Zealand and is associated with significant treatment and societal costs. Clinical practice guidelines (CPGs) for LBP increasingly recommend the use of screening and stratification tools to aid the early identification of psychosocial factors that can contribute to chronic LBP. This survey of New Zealand physiotherapists examined their use of CPGs, screening, and stratification tools in clinical practice, and identified their perceived barriers to using these tools. In total, 228 physiotherapists completed the survey. Over half of the respondents (53\%) regularly used CPGs for LBP in clinical practice, with the Accident Compensation Corporation's New Zealand Acute Low Back Pain Guide being the most commonly used guideline (84\%). Most (94\%) respondents reported screening people with LBP for psychosocial factors; $37 \%$ used formal screening tools and $22 \%$ used risk stratification tools. Key perceived barriers to using CPGs, screening, and stratification tools included lack of training and exposure, time constraints, and lack of resources. An analysis using chi-square tests revealed significant associations ( $p<0.05)$ between the use of screening tools, and postgraduate qualifications and years of experience. Further research is required to better understand whether a stratified model of care for LBP may be implemented in New Zealand and the supports required to ensure the success of such a model.

Hill, J., Bedford J., Houston, D., Reid, D. A., Baxter, G. D., \& Ellis, R. (2020). Exploring physiotherapists' use of clinical practice guidelines, screening, and stratification tools for people with low back pain in New Zealand. New Zealand Journal of Physiotherapy, 48(2), 59-69. https://doi.org/10.15619/NZJP/48.2.02

Key Words: Low Back Pain, Physiotherapy, Stratified Care, Clinical Guidelines, Screening Tools

\section{INTRODUCTION}

Spinal disorders are the leading specific cause of health loss (as measured by disability adjusted life years) for those aged 15-64 years in New Zealand (Ministry of Health, 2016). The prognosis for acute low back pain (LBP) is generally positive, with pain and disability often improving within 6 weeks of onset (Artus et al., 2014; Green et al., 2018; Menezes Costa et al., 2012). However, a review of prospective studies investigating the prognosis of recent onset LBP reported that $65 \%$ of people were still experiencing LBP at their 12-month follow-up appointment, indicating that the prognosis is often not as favourable as suggested in clinical guidelines (Itz et al., 2013). Moreover, the traditional concept of LBP as discreet, unrelated episodes has been challenged, and LBP is increasingly considered a longlasting condition with a variable course (Dunn et al., 2013). This pattern of recurrence and the disability that ensues in some cases of chronic LBP may be explained by the complex interrelationship of biomedical, psychological, and social factors that can contribute to LBP (Foster \& Delitto, 2011; O'Sullivan et al., 2016; Ramond et al., 2011).

Treatment expenditure for LBP is increasing, and much of this cost is absorbed by the disability related to chronic LBP. It is estimated that $80 \%$ of direct public healthcare expenditure in New Zealand relates to chronic LBP (National Health Committee, 
2015). Further, costs associated with loss of income and productivity attributable to LBP have been estimated at $\$ 2.6$ billion (National Health Committee, 2015). These estimates demonstrate the substantial societal costs of chronic LBP and highlight the importance of early identification of people who are at risk of developing a disability related to chronic LBP.

Clinical practice guidelines (CPGs) are formal, evidence-based recommendations that seek to optimise health outcomes and are considered fundamental to improving health care (Lin et al., 2019). Early physiotherapy treatment that adheres to CPGs for LBP has been shown to significantly reduce the use of imaging, lumbar injections, surgery, and opioids as well as reducing total treatment costs (Childs et al., 2015). CPGs increasingly recommend using validated prognostic screening tools to help identify psychosocial factors, often referred to as "yellow flags", and guide the management of LBP (Oliveira et al., 2018). The Accident Compensation Corporation (ACC) published the latest edition of the New Zealand Acute Low Back Pain Guide in 2004, which covered the assessment and management of acute LBP. This guideline recommends administering the Örebro Musculoskeletal Pain Screening Questionnaire (ÖMPSQ) if patients do not make the expected progress in the first 2-4 weeks (Accident Compensation Corporation, 2004).

Stratified care targets treatment based on biological or other prognostic risk factors that are evident in subgroups of patients (Hingorani et al., 2013). This supports clinical decision-making and increases the efficiency of healthcare provision while maximising patient outcomes (Foster et al., 2013; Hingorani et al., 2013). Validated prognostic screening tools are available that assess a combination of factors and help predict future outcomes; such tools are integral to stratified care (Steyerberg et al., 2013). LBP is considered well suited to stratified care because of the heterogenous populations, large variations in prognosis, and the multitude of treatment options that have varying risks and costs (Foster et al., 2013; Hodges, 2019). There are three broad approaches to stratified care for LBP. These are based on: 1) underlying mechanisms, 2) treatment responsiveness, and 3) risk for persistent disability (Foster et al., 2013). The United Kingdom National Institute for Health and Care Excellence (NICE) guidelines for LBP recommend the use of risk stratification tools, such as the STarT Back Screening Tool (SBST), at the first contact for each new LBP episode (National Institute for Health and Care Excellence [NICE], 2016). In the UK primary care context, use of the SBST resulted in reduced levels of disability, increased health-related quality of life, and cost savings compared with usual care (Foster et al., 2014; Hill et al., 2011)

Several studies have investigated the degree to which New Zealand physiotherapists use CPGs for LBP. Tumilty et al. (2017) reviewed treatment records from private physiotherapy clinics in New Zealand and found that despite reducing pain and improving function, the most commonly applied treatments (e.g. joint mobilisations, specific exercises, and massage) lacked support from CPGs. A survey of New Zealand physiotherapists by Hendrick et al. (2013) found that although the majority of respondents provided advice consistent with CPGs (e.g. returning to activity and work, and avoiding bedrest), adherence to CPGs was influenced by the therapists' level of education and the extent of their biomedical beliefs. An earlier study by Copeland et al. (2008) found that the use of LBP outcome measures by New Zealand physiotherapists was relatively low $(40 \%)$, although that study did not include screening or stratification tools, such as the ÖMPSQ or SBST.

To date, no research has investigated the extent to which (and how) screening and stratification tools are used by physiotherapists in clinical practice for the assessment and management of people with LBP in New Zealand. Furthermore, if there are barriers to physiotherapists using these tools, these barriers have not been clearly identified. Therefore, the aim of this study was to survey registered physiotherapists practicing in New Zealand who regularly treat people with LBP to investigate:

1. The extent to which New Zealand physiotherapists use CPGs, screening, or stratification tools (collectively, "the tools") in assessing and treating people with LBP.

2. For those physiotherapists using the tools, which tools are being used, how they are used to assess/treat people with LBP, and their perceived importance.

3. For those physiotherapists not using the tools, the perceived barriers to using the tools.

\section{METHODS}

This study used a cross-sectional observational design, with data gathered through an internet-based survey of New Zealand registered physiotherapists. The survey comprised three main phases: 1) survey development, 2) face validity testing through expert consultation, and 3) survey distribution and data collection.

\section{Survey development}

The 39-item survey contained four sections: 1) participants' consent and professional background, 2) CPGs, 3) screening tools, and 4) stratification tools. For the purposes of this study, we defined general LBP questionnaires/outcome measures, such as the Oswestry Low Back Pain Disability Questionnaire, as screening tools, because these tools can be used by clinicians to identify people at risk of chronicity through to slow recovery or poor outcomes. Although the Oswestry Low Back Pain Disability Questionnaire and similar tools were not specifically designed as screening and/or stratification tools, they help practitioners in their decision-making about treatment pathways.

The last three sections of this study followed a similar pattern of assessing individual selection of the tools, exploring how the tools guided clinical practice, and where relevant, any associated barriers to the use of the tools.

Face validity testing via an expert panel

The face validity of the survey was assessed by a panel of four experts in the field of assessment and treatment of people with LBP. As the survey aimed to understand the New Zealand context, three of these experts were based in New Zealand. The fourth expert was from the UK to provide an international perspective.

The survey initially combined screening and stratification tools in the same section. However, following feedback from the panel, these types of tools were separated to allow clarity between screening for psychosocial barriers and stratification into 
treatment pathways. Other modifications included removing questions or response options deemed not applicable and adding the option to provide qualitative explanations for certain items.

\section{Survey distribution and data collection}

The final survey was distributed through SurveyMonkey®, an internet-based survey site. Participation in the survey was anonymous and no identifying data were collected from participants. The survey was advertised via newsletters, clinical meetings, and relevant social media platforms operated by Physiotherapy New Zealand and its associated special interest groups. Participant recruitment was enhanced by a snowballing method, whereby participants were encouraged to promote the survey to other physiotherapists. Eligibility to participate in the survey was limited to physiotherapists registered in New Zealand who regularly assess and treat people with LBP. Ethical approval to conduct this study was obtained from the Auckland University of Technology Ethics Committee (reference number 19/72). The survey was open for responses from 10 April to 23 September 2019. All participants provided consent to participate in the study.
Data analysis

Data were exported from SurveyMonkey®and analysed using SPSS version 23.0 (IBM Corp., Armonk, NY, USA). Data for closed or multiple-choice questions were presented as frequencies and percentages, based on the number of valid responses per item. For the open-ended questions, two researchers (JB and $\mathrm{DH})$ independently reviewed all responses. Through consensus agreement, they grouped similar responses into like categories, and then frequencies and percentages were calculated for the valid responses in each category. Non-parametric chi-square analyses were used to compare demographic and professional details of physiotherapists who used CPGs, screening, and stratification tools (termed "users") with physiotherapists who did not use these tools (termed "nonusers"). The level of significance was set at $p<0.05$.

\section{RESULTS}

\section{Survey responses}

Of the 300 participants who responded to the survey, 17 did not meet the inclusion criteria (Figure 1). A further 55 participants were excluded because they did not complete the survey in full. This left complete responses from 228 participants for inclusion in the analysis.

\section{Figure 1}

Prototypical STARD Diagram of the Flow of Participants Through the Study

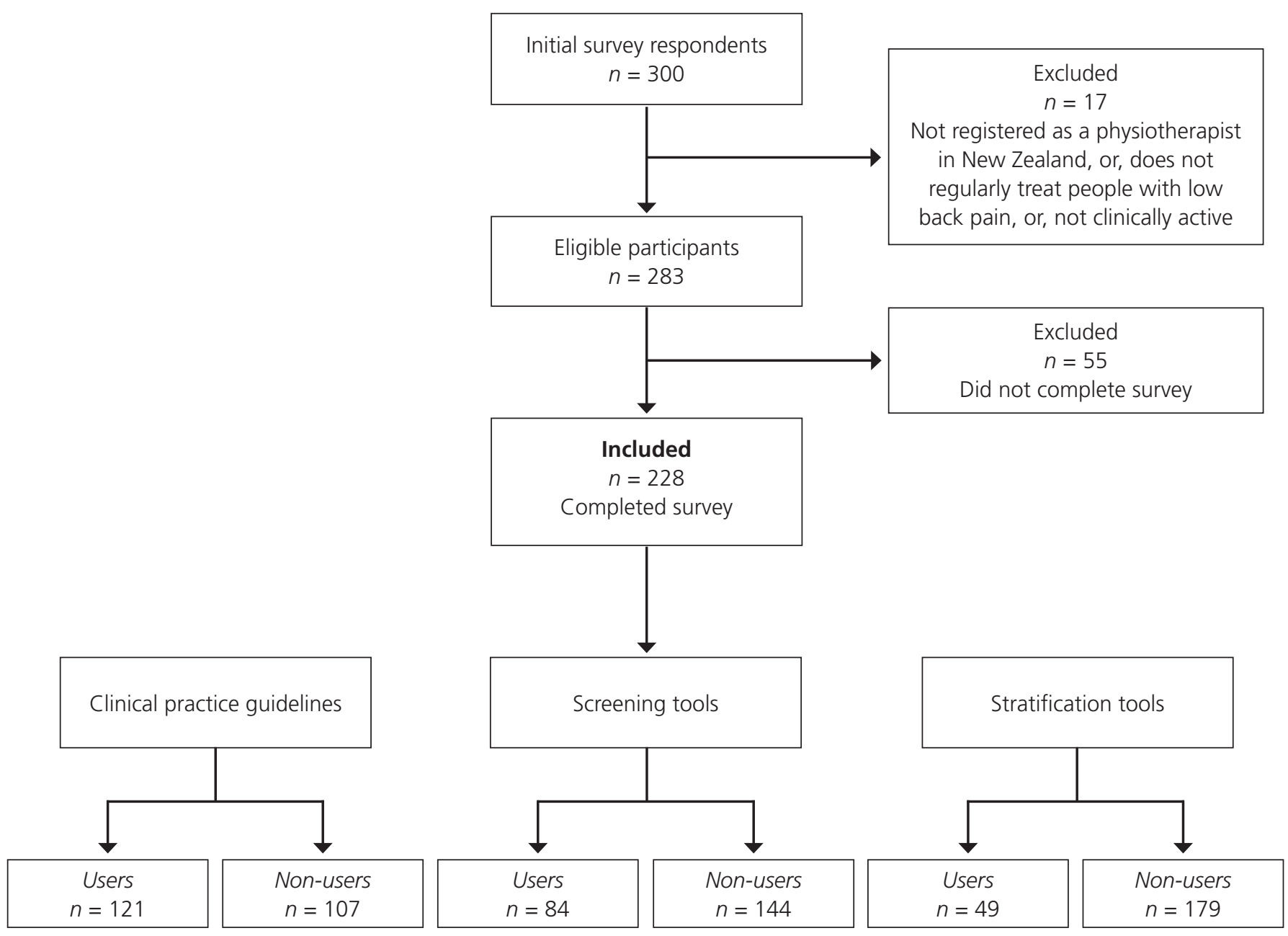


Professional and demographic details

Over half of the participants were aged 20-39 years (138/228; $61 \%)$, with $47 \%(107 / 228)$ having $\leq 10$ years of clinical experience (Table 1). A graduate degree in physiotherapy was the highest qualification for $47 \%$ (108/228) of participants, and the majority of participants had obtained their qualification from New Zealand institutions (184/228; $81 \%$ ). The survey allowed participants to select multiple areas and settings of clinical practice; most participants indicated that they worked in musculoskeletal physiotherapy $(218 / 228 ; 96 \%)$ and in a private practice setting (201/228; 88\%) (Table 1).

\section{Use of CPGs}

Over half of the participants reported regular use of CPGs during their assessment and treatment of people with LBP $(121 / 228 ; 53 \%)$. The mean importance rating for use of CPGs was 6 out of 10 (zero = "not at all important" and $10=$ "very important"). The most frequently used CPGs were the New Zealand Acute Low Back Pain Guide (101/121; 84\%) and the NICE Guidelines (30/121; 25\%) (Table 2). Aspects of CPGs that were most commonly used in the assessment of people with LBP included screening for red flags $(98 / 121 ; 81 \%)$, guidance on referral for diagnostic imaging $(79 / 121 ; 65 \%)$, and screening for yellow flags $(69 / 121 ; 57 \%)$. The components of CPGs most commonly used to guide treatment methods were guidance on conservative treatment modalities $(84 / 121 ; 69 \%)$, onwards referral to other treatment modalities $(59 / 121 ; 49 \%)$, and referral for surgery $(61 / 121 ; 50 \%)$.

Of the participants that reported they did not use CPGs regularly $(107 / 228 ; 47 \%)$, the main reasons identified were a lack of necessity due to personal knowledge and training (32/107; $30 \%)$, no training in or exposure to the guidelines (35/107; $33 \%)$, the lack of relevance to rehabilitation pathways (19/107; $18 \%)$, and that guidelines were out of date/not evidence based $(16 / 107 ; 15 \%)$ (Table 3$)$. The most commonly reported ways to potentially reduce barriers to using CPGs included attending informal training courses $(72 / 107 ; 67 \%)$ and updating the applicable clinical guidelines (50/107; 47\%) (Table 4).

\section{Table 1}

Participant Demographics by use of Clinical Practice Guidelines, Screening and Stratification Tools: Users Versus Non-users

\begin{tabular}{|c|c|c|c|c|c|c|c|}
\hline \multirow{2}{*}{ Participant demographics } & \multicolumn{3}{|c|}{ CPGs } & \multicolumn{2}{|c|}{ Screening tools } & \multicolumn{2}{|c|}{ Stratification tools } \\
\hline & & & & $n(\%)$ & & & \\
\hline \multicolumn{8}{|l|}{ Age (years) } \\
\hline $20-29$ & $69(30.3)$ & $43(35.5)$ & $26(24.3)$ & $22(26.2)$ & $47(32.6)$ & $1326.5)$ & $56(31.3)$ \\
\hline $30-39$ & $69(30.3)$ & $29(24.0)$ & $40(37.4)$ & $29(34.5)$ & $40(27.8)$ & $1632.7)$ & $53(29.6)$ \\
\hline $40-49$ & $46(20.2)$ & $25(20.7)$ & $21(19.6)$ & $19(22.6)$ & $27(18.8)$ & $1122.4)$ & $35(19.6)$ \\
\hline $50-59$ & $29(12.7)$ & $16(13.2)$ & $13(12.1)$ & $8(9.5)$ & $21(14.6)$ & $6(12.2)$ & $23(12.8)$ \\
\hline $60-69$ & $15(6.6)$ & $8(6.6)$ & $7(6.5)$ & $6(7.1)$ & $9(6.3)$ & $3(6.1)$ & $12(6.7)$ \\
\hline $70+$ & $0(0)$ & $0(0)$ & $0(0)$ & $0(0)$ & $0(0)$ & $0(0)$ & $0(0)$ \\
\hline \multicolumn{8}{|l|}{ Work experience (years) } \\
\hline $0-5$ & $70(30.7)$ & $39(32.2)$ & $31(29.0)$ & $15(17.9)$ & $55(38.2)$ & $13(26.5)$ & $57(31.8)$ \\
\hline $6-10$ & $37(16.2)$ & $23(19.0)$ & $14(13.1)$ & $20(23.8)$ & $17(11.8)$ & $12(24.5)$ & $25(14.0)$ \\
\hline $11-15$ & $40(17.5)$ & $17(14.0)$ & $23(21.5)$ & $16(19.0)$ & $24(16.7)$ & $9(18.4)$ & $31(17.3)$ \\
\hline $16+$ & $81(35.5)$ & $42(34.7)$ & $39(36.4)$ & $33(39.3)$ & $48(33.3)$ & $15(30.6)$ & 66 (36.9) \\
\hline \multicolumn{8}{|l|}{ Further qualification } \\
\hline None & $108(47.4)$ & $53(43.8)$ & $55(51.4)$ & $28(33.3)$ & $80(55.6)$ & $17(34.7)$ & $91(50.8)$ \\
\hline Postgraduate & $76(33.3)$ & $42(34.7)$ & $34(31.8)$ & $32(38.1)$ & $44(30.6)$ & $17(34.7)$ & $59(33.0)$ \\
\hline Master's & $41(18.0)$ & $23(19.0)$ & $18(16.8)$ & $22(26.2)$ & $19(13.2)$ & $14(28.6)$ & $27(15.1)$ \\
\hline Other & $3(1.3)$ & $3(2.5)$ & $0(0)$ & $2(2.4)$ & $1(0.7)$ & $1(2.0)$ & $2(1.1)$ \\
\hline \multicolumn{8}{|l|}{ Area(s) of work } \\
\hline Musculoskeletal & $218(95.6)$ & $115(95.0)$ & $103(96.3)$ & $79(94.0)$ & $139(96.5)$ & $45(91.8)$ & $173(96.6)$ \\
\hline Sports physiotherapy & $105(46.1)$ & $59(48.8)$ & $46(43.0)$ & $37(44.0)$ & $68(47.2)$ & $18(36.7)$ & $87(48.6)$ \\
\hline Multidisciplinary & $36(15.8)$ & $19(15.7)$ & $17(15.9)$ & $29(34.5)$ & $7(4.9)$ & $14(28.6)$ & $22(12.3)$ \\
\hline Occupational health & $25(11.0)$ & $18(14.9)$ & $7(6.5)$ & $15(17.9)$ & $10(6.9)$ & $8(16.3)$ & $17(9.5)$ \\
\hline Other & $27(11.8)$ & $14(11.6)$ & $13(12.1)$ & $8(9.5)$ & $19(13.2)$ & $4(8.2)$ & $23(12.8)$ \\
\hline \multicolumn{8}{|l|}{ Setting(s) of work } \\
\hline Public hospital & $22(9.6)$ & $13(10.7)$ & $9(8.4)$ & $10(11.9)$ & $12(8.3)$ & $5(10.2)$ & $17(9.5)$ \\
\hline Private practice & $201(88.2)$ & $104(86.0)$ & $97(90.7)$ & $71(84.5)$ & $130(90.3)$ & $41(83.7)$ & $160(89.4)$ \\
\hline Private organisation & $7(3.1)$ & $3(2.5)$ & $4(3.7)$ & $3(3.6)$ & $4(2.8)$ & $3(6.1)$ & $4(2.2)$ \\
\hline Sports institute & $40(17.5)$ & $26(21.5)$ & $14(13.1)$ & $10(11.9)$ & $30(20.8)$ & $6(12.2)$ & $34(19.0)$ \\
\hline Other & $8(3.5)$ & $3(2.5)$ & $5(4.7)$ & $1(1.2)$ & $7(4.9)$ & $0(0)$ & $8(4.5)$ \\
\hline
\end{tabular}

Note. CPGs = clinical practice guidelines. 
Table 2

Clinical Practice Guidelines Used in Clinical Practice

\begin{tabular}{lc}
\hline Clinical practice guidelines & $n(\%)$ \\
\hline New Zealand acute low back pain guide (Accident Compensation Corporation) & 101 (83.5) \\
Low back pain and sciatica in over 16s: Assessment and management (National Institute for & 30 (24.8) \\
Health and Care Excellence guideline, UK) & $14(11.6)$ \\
Management of non-specific back pain and lumbar radicular pain (Best Practice Advocacy Centre & $12(9.9)$ \\
New Zealand [BPACNZ]) & $12(9.9)$ \\
Acute low back pain (Best Practice Advocacy Centre New Zealand [BPACNZ]) & 6 (5.0) \\
Diagnosis and treatment of low back pain: A joint clinical practice guideline (American College of \\
Physicians and the American Pain Society, USA) \\
Guideline for the evidence-informed primary care management of low back pain (College of \\
Family Physicians, Canada) \\
Low back pain: Clinical practice guidelines linked to the International Classification of \\
Functioning, Disability and Health (Orthopaedic Section of the American Physical Therapy \\
Association, USA) \\
Other
\end{tabular}

Note. $N=121$

\section{Table 3}

Barriers for not Using Clinical Practice Guidelines, Screening and Stratification Tools

\begin{tabular}{|c|c|c|c|}
\hline \multirow[t]{2}{*}{ Barriers } & $\begin{array}{c}\text { CPGs } \\
(n=107)\end{array}$ & $\begin{array}{l}\text { Screening tools } \\
\quad(n=13)\end{array}$ & $\begin{array}{l}\text { Stratification tools } \\
\qquad(n=179)\end{array}$ \\
\hline & \multicolumn{3}{|c|}{$n(\%)$} \\
\hline No interest & $4(3.7)$ & $5(3.8)$ & $14(7.8)$ \\
\hline No training or exposure & $35(32.7)$ & $32(24.4)$ & $123(68.7)$ \\
\hline I don't understand the potential use & $13(12.1)$ & $10(7.6)$ & $39(21.8)$ \\
\hline No need due to personal knowledge & $32(29.9)$ & $15(11.5)$ & $19(10.6)$ \\
\hline Out of date/not evidence based & $16(15.0)$ & N/A & N/A \\
\hline Not individualised to patient & $4(3.7)$ & $3(2.3)$ & $4(2.4)$ \\
\hline Don't feel competent despite recieving training & $4(3.7)$ & $5(3.8)$ & $10(5.6)$ \\
\hline Lack of support from management & $12(11.2)$ & $18(13.7)$ & $21(11.7)$ \\
\hline Lack of confidence & $7(6.5)$ & $16(12.2)$ & $17(9.5)$ \\
\hline Lack of relevance to rehabilitation pathway & $19(17.8)$ & $16(12.2)$ & $15(8.4)$ \\
\hline I am aware of them but don't use them & $11(10.3)$ & N/A & N/A \\
\hline Lack of awareness/not front of mind & N/A & $6(4.6)$ & $2(1.2)$ \\
\hline Lack of resources to administer and collate data & N/A & $36(27.5)$ & $33(18.4)$ \\
\hline Patients not willing to complete & N/A & $19(14.5)$ & $9(5.0)$ \\
\hline Time constraints & N/A & $86(65.6)$ & $61(34.1)$ \\
\hline Other & $2(1.8)$ & $5(3.8)$ & $8(4.5)$ \\
\hline
\end{tabular}

Note. $\mathrm{CPGs}=$ clinical practice guidelines; N/A = not applicable. Participants able to select more than one option . 


\section{Table 4}

Potential Ways to Reduce Barriers to Using Clinical Practice Guidelines, Screening and Stratification Tools

\begin{tabular}{|c|c|c|c|}
\hline \multirow[t]{2}{*}{ Ways to reduce barriers } & $\begin{array}{c}\text { CPGs } \\
(n=107)\end{array}$ & $\begin{array}{l}\text { Screening tools } \\
\quad(n=131)\end{array}$ & $\begin{array}{c}\text { Stratification tools } \\
\qquad(n=179)\end{array}$ \\
\hline & \multicolumn{3}{|c|}{$n(\%)$} \\
\hline Attending formal training courses & $36(33.6)$ & $30(22.9)$ & $55(30.7)$ \\
\hline Attending informal training courses & $72(67.3)$ & $62(47.3)$ & $117(65.4)$ \\
\hline Use of "clinical champions" to promote use & $19(17.8)$ & $21(16.0)$ & $27(15.1)$ \\
\hline Profesional body engagement and endorsement & $37(34.6)$ & $26(19.8)$ & $45(25.1)$ \\
\hline Funding providers mandating use & $15(14.0)$ & $25(19.1)$ & $34(19.0)$ \\
\hline Update of applicable clinical guidelines & $50(46.7)$ & N/A & $\mathrm{N} / \mathrm{A}$ \\
\hline Longer patient appointment times & N/A & $61(46.6)$ & $51(28.5)$ \\
\hline More assistance to administer and collate data & $\mathrm{N} / \mathrm{A}$ & $61(46.6)$ & $58(32.4)$ \\
\hline Further evidence to support use & $3(2.8)$ & $41(31.3)$ & $58(32.4)$ \\
\hline Other & $6(5.6)$ & $5(3.8)$ & $11(6.1)$ \\
\hline
\end{tabular}

Note. $\mathrm{CPGs}=$ clinical practice guidelines; N/A = not applicable .

\section{Use of screening tools}

Regular screening for yellow flags and psychosocial factors in people with LBP was reported by most participants (215/228; $94 \%)$. Of these participants, approximately two-thirds did not use formal questionnaires or screening tools (131/215; 61\%). Other ways that participants reported incorporating screening into assessment included targeted questions within the subjective interview (197/215; 92\%), screening based on previous history of pain and disability $(116 / 215 ; 54 \%)$, and the patient not improving within expected timeframes (103/215; 48\%).

The most commonly used screening tools were the ÖMPSQ - Short Form and the Oswestry Low Back Pain Disability Questionnaire (Table 5). Of the screening tool users (84/215; $39 \%), 60 \%$ (50/84) reported being selective when incorporating these tools in assessment (i.e. based on clinical reasoning rather than for every patient). Common factors that influenced participants' decisions to administer screening tools were indicators of low mood (47/50; 94\%) and the patient's responses in the subjective interview $(41 / 50 ; 82 \%)$. Other indicators were past history of chronic pain or disability (32/50; $64 \%)$, past history of LBP $(16 / 50 ; 32 \%)$, and if the patient did not show improvements within an expected timeframe (28/50; $56 \%$ ). Respondents were also asked to report on the purpose, situational context, and how they were introduced to using screening tools (Table 6). The mean score for the importance of screening tools in informing the assessment and treatment of people with LBP was 7 out of 10.

The non-users of screening tools (131/215; 61\%) were asked to identify barriers preventing the use of these tools (Table 3) and to suggest potential ways to reduce these perceived barriers (Table 4). Common barriers included time constraints $(86 / 131 ; 66 \%)$, lack of resources to administer and collate data $(36 / 131 ; 28 \%)$, and lack of training (32/131; $24 \%)$. Common suggestions for reducing barriers included attending informal training courses $(62 / 131 ; 47 \%)$, assistance to administer and collate data $(61 / 131 ; 47 \%)$, longer patient appointment times $(61 / 131 ; 47 \%)$, and further evidence to support the use of screening tools $(41 / 131 ; 31 \%)$.

Use of stratification tools

Regular clinical use of stratification tools was reported by $22 \%$ of participants (49/228), with the most commonly used tools being the SBST (28/49; 57\%) and ÖMPSQ - Short Form (27/49; 55\%) (Table 7). The mean score for the importance of stratification tools was 7 out of 10 . Most commonly, participants reported that these tools were used as needed, based on clinical judgment (22/49; 45\%). The majority of stratification tool users reported the purpose of using these tools was to inform the treatment approach $(41 / 49 ; 84 \%)$ and inform the need for an escalated level of treatment management (38/49; 78\%). Other reasons included monitoring treatment progress and recovery (26/49; 53\%), and informing subjective and/or objective assessment (23/49; 47\%) (Table 6).

The majority of participants $(179 / 228 ; 79 \%)$ reported that they did not use stratification tools on a regular basis. The most common perceived barriers were no training (123/179; 69\%), time constraints $(61 / 179 ; 34 \%)$, not understanding the potential uses in clinical practice $(39 / 179 ; 22 \%)$, and lack of resources to administer and collate data (33/179; 18\%) (Table 3). Potential ways to reduce barriers included attending informal training courses $(117 / 179 ; 65 \%)$, more assistance to administer and collate data $(58 / 179 ; 32 \%)$ (Table 4$)$, further evidence to support the use of stratification tools $(58 / 179 ; 32 \%)$, and longer patient appointment times (51/179; 29\%).

The results of the chi-square analyses (Table 8) showed there were significant associations between the use of screening tools and participants' level of education and years of work experience. No significant associations were found for the use of CPGs or stratification tools. However, there was a significant association between participants who used CPGs and those who used stratification tools. 
Table 5

Screening Tools and Outcome Measures Used in Clinical Practice

\begin{tabular}{lr}
\hline Screening tools and outcome measures & $n(\%)$ \\
\hline Örebro Musculoskeletal Pain Questionnaire (ÖMPQ) - Short Form & $52(61.9)$ \\
Oswestry Low Back Pain Disability Questionnaire & $39(46.3)$ \\
Depression, Anxiety and Stress Scales (DASS-21) & $33(39.3)$ \\
Pain Catastrophising Scale & $33(39.3)$ \\
Pain Self-Efficacy Questionnaire (PSEQ) & $31(36.9)$ \\
STarT Back Screening Tool (SBST) & $26(31.0)$ \\
electronic Persistant Pain Outcomes Collaboration (ePPOC) & $25(29.8)$ \\
Tampa Scale for Kinesiophobia (TSK) & $20(23.8)$ \\
Roland-Morris Low Back Pain and Disability Questionnaire (RMQ) & $19(22.6)$ \\
Fear Avoidance Beliefs Questionnaire (FABQ) & $18(21.4)$ \\
Örebro Musculoskeletal Pain Questionnaire (ÖMPQ) - Long Form & $10(11.9)$ \\
Short Form-36 (SF-36) & $10(11.9)$ \\
Central Sensitisation Inventory & $9(10.7)$ \\
Back Pain Inventory (BPI) & 5 (6.0) \\
Hospital Anxiety and Depression Questionnaire (HADs) & 5 (6.0) \\
Other & $12(14.3)$
\end{tabular}

Note. $N=84$.

\section{Table 6}

Purpose, Situational Context and Introduction to Screening and Stratification Tools

Variable

$\begin{array}{cc}\begin{array}{c}\text { Screening tools } \\ (n=84)\end{array} & \begin{array}{c}\text { Stratification tools } \\ (n=49)\end{array} \\ n(\%)\end{array}$

Purpose

To inform the subjective and/or objective assessment

$38(45.2)$

$23(46.9)$

To inform treatment approach

$59(70.2)$

$41(83.7)$

To monitor treatment progress and recovery

$61(72.6)$

$26(53.1)$

To inform the need for an escalated level of treatment management

$68(81.0)$

$38(77.6)$

Research

$6(7.1)$

$3(6.1)$

Situation

During first consultation only

$11(13.1)$

$11(22.4)$

Every consultation

$2(2.4)$

$3(6.1)$

During first and final consultation only

$25(29.8)$

$13(26.5)$

Sporadically/as needed based on clinical judgment

$43(51.2)$

$22(44.9)$

Other

$3(3.6)$

$0(0)$

Introduction to tools

Included in university degree

Formal training course

$42(50.0)$

$0(0)$

$19(38.8)$

Informal training course

$33(39.3)$

$0(0)$

Personal ongoing professional development

39 (46.4)

$17(34.7)$

Clinical requirement of current/previous employer

$19(38.8)$

$31(36.9)$

$15(30.6)$

Clinical requirement of a treatment provider

42 (50.0)

$13(26.5)$

Reccomended by a colleague

17 (20.2)

$9(18.4)$

Knowledge of clinical guidelines

$23(27.4)$

$9(18.4)$

Other

$4(4.8)$

$0(0)$ 
Table 7

Stratification Tools and Outcome Measures Used in Clinical Practice

\begin{tabular}{lc}
\hline Stratification tools and outcome measure & $n(\%)$ \\
\hline STarT Back Screening Tool (SBST) & $28(57.1)$ \\
Örebro Musculoskeletal Pain Questionnaire (ÖMPQ) - Short Form & $27(55.1)$ \\
electronic Persistant Pain Outcomes Collaboration (ePPOC) & $12(24.5)$ \\
Örebro Musculoskeletal Pain Questionnaire (ÖMPQ) - Long Form & $6(12.2)$ \\
Other & $6(12.2)$ \\
\hline
\end{tabular}

Note. $N=49$.

Table 8

Associations Between Participants' Level of Education and Years of Work Experience With the Use of Screening Tools

\begin{tabular}{lllc}
\hline & Variable & & \\
\hline Variable 1 & Variable 2 & Statistic & $p$ value \\
\hline Postgraduate vs. non-postgraduate & CPG user vs. non-user & $\chi^{2}(1)=1.028$ & 0.311 \\
Postgraduate vs. non-postgraduate & Screening tool user vs. non-user & $\chi^{2}(1)=9.636$ & $0.002^{*}$ \\
Postgraduate vs. non-postgraduate & Stratification tool user vs. non-user & $\chi^{2}(1)=3.400$ & 0.065 \\
Years of experience & CPG user vs. non-user & $\chi^{2}(3)=3.267$ & 0.352 \\
Years of experience & Screening tool user vs. non-user & $\chi^{2}(3)=12.558$ & $0.006^{*}$ \\
Years of experience & Stratification tool user vs. non-user & $\chi^{2}(3)=3.427$ & 0.330 \\
CPG user vs. non-user & Screening tool user vs. non-user & $\chi^{2}(1)=2.653$ & 0.103 \\
CPG user vs. non-user & Stratification tool user vs. non-user & $\chi^{2}(1)=7.533$ & $0.006^{*}$ \\
\hline
\end{tabular}

Note. $\mathrm{CPG}=$ clinical practice guideline.

${ }^{*} p<0.05$.

\section{DISCUSSION}

The 228 complete surveys included in this analysis represented approximately $4.3 \%$ of the estimated 5,346 physiotherapists in New Zealand who held an Annual Practicing Certificate (APC) in 2019 , or $7.3 \%$ if only APC holders who worked in private practice were included (Physiotherapy Board of New Zealand, 2019). Of all participants, $60.6 \%$ were aged $\leq 39$ years, and $30.3 \%$ were aged $\leq 29$ years. This is broadly comparable with the average age (36.4 years) of New Zealand registered physiotherapists employed in private practice (excluding those who were self-employed) (Physiotherapy Board of New Zealand, 2019). However, our participants were notably younger compared with the paper-based survey on LBP outcome measures mailed to practice owners by Copeland et al. (2008). The younger demographic in this study may be attributable to the survey being electronic and promoted via social media platforms, which often attracts younger respondents compared with mailed surveys or other recruitment methods (Dykema et al., 2013; Topolovec-Vranic \& Natarajan, 2016).

The qualification level among our participants was higher than that of New Zealand registered physiotherapists overall, with $53 \%$ of our participants holding a postgraduate qualification compared with $45 \%$ of the wider physiotherapy population (Physiotherapy Board of New Zealand, 2019). The majority of participants in this study worked in private practice (88\%), compared with $58 \%$ of all 2019 APC holders. However, this higher proportion was expected given the survey's focus on LBP, which is commonly treated in private practice. Our participants were comparable with those of the previous survey by Hendrick et al. (2013), which investigated New Zealand physiotherapists' knowledge and use of CPGs for LBP (92\% in private practice).

The survey found that $53 \%$ of participants regularly used CPGs in their practice, which was consistent with the $52 \%$ reported by Hendrick et al. (2013). A lack of training and exposure to CPGs was perceived as a major barrier to using CPGs. Often, participants used their clinical experience and individualised approach guided their assessment and treatment of patients, rather than using CPGs. Both this identified barrier and the proportion of regular users in our study were consistent with a survey by Bernhardsson et al. (2014) that investigated the determinants of the use of CPGs by Swedish physiotherapists. That study found that $47 \%$ of respondents frequently used CPGs but also identified lack of time to refer to CPGs (68\%) and uncertainty on how to access them (45\%) as key barriers to the use of CPGs. Participants in this study indicated that attending formal or informal training courses and endorsement by professional organisations may reduce the barriers to the use of CPGs. Of the non-users, $47 \%$ believed that updating the guidelines with current evidence would encourage greater use of CPGs. 
Several participants emphasised how patients' treatment expectations and biomedical beliefs about LBP dictated the treatment approach adopted, which was often not supported by CPGs. However, some of these clinicians may have had biomedical beliefs and perspectives, which have previously been shown to influence patients' expectations, and the treatment approach and education that clinicians provide to their patients (Darlow et al., 2012; Hendrick et al., 2013). Large-scale public education programmes have been implemented in several countries to change patient beliefs about LBP. However, these programmes have largely been unsuccessful, possibly because of the reinforcement of biomedical perspectives by health practitioners (Zusman, 2013).

Almost all participants in this study indicated that they screened patients for yellow flags, but the methods used lacked consistency. Although 37\% of participants reported using formal screening tools, $51 \%$ of these participants indicated they used clinical judgement to guide when and with whom they were used, rather than routinely using screening tools with all patients. The interrater agreement between expert clinicians' clinical judgement for patient risk allocation and the risk allocation determined by the SBST has been shown to be "fair" (Hill et al., 2010; Miki et al., 2020). Therefore, this inconsistency in patient screening is of concern and shows that clinical judgement is probably not the best basis for deciding when/if to screen for psychosocial risk factors (Miki et al., 2020). A systematic review of qualitative studies by Synnott et al. (2015) found that although physiotherapists recognised psychosocial factors in LBP patients, they preferred to treat the mechanical aspects of LBP and may stigmatise people based on psychosocial factors. Furthermore, physiotherapists often indicated they lacked the training and skillset to effectively address psychosocial factors in clinical practice (Karstens et al., 2018; Synnott et al., 2015).

Stratification tools, such as the SBST, aim to identify subgroups of patients and support clinical decision-making, thereby reducing harms, increasing the efficiency of healthcare provision, and maximising patient outcomes. Stratification is about ensuring appropriate matched treatment for all subgroups, not just the high-risk subgroup of patients. A key finding of this study was that only $21 \%$ of participants used stratification tools in clinical practice. This was unsurprising, as risk-based stratification is a relatively new area of focus in LBP research and few formal acute treatment pathways currently exist for high-risk patients in New Zealand. Notably, of those who reported using stratification tools, $45 \%$ used them in a sporadic/inconsistent manner based on their clinical judgement. This indicated that the use of stratification tools was often not consistent with the way they were designed and validated. This barrier to the correct implementation of stratification tools may be attributed to a lack of training. Time constraints and lack of resources to administer and collate results from stratification and screening tools were other identified barriers. This was consistent with the previous survey investigating the use of LBP outcome measures by Copeland et al. (2008), which found that private practitioners often felt pressured to see as many patients as possible to stay financially viable, with the additional time required to use outcome measures not being remunerated by funders. This could potentially be a barrier to implementing tools into practice that stratify patients into a category that means they receive less treatment than they may have done without stratification. Some patient's treatment is funded by ACC and some is not. This has the potential to also affect a patient's choices for treatment practitioner. Concern about lack of financial incentives to adopt stratified care for LBP was identified in a qualitative study of German physiotherapists (Karstens et al., 2018), which also reported participants felt they did not have the necessary skills to deliver psychosocially informed treatment. Given these challenges, it is likely that the adoption of a stratified model of care for LBP requires an approach tailored to each country to ensure it is fit for purpose and acceptable to multiple stakeholders (Sowden et al., 2018).

Study limitations

This study had several limitations. Firstly, the use of an Internetbased survey promoted via multiple platforms and organisations meant it was not possible to calculate a response rate at the onset. Although participants' demographics were broadly representative of the target population, the 228 complete responses represented approximately $4.3 \%$ of all potential participants, which may limit the generalisability of the results. Secondly, the results and demographics of this survey may have been impacted by self-selection bias, which is a recognised disadvantage of online surveys (Khazaal et al., 2014). For example, recent graduates might have had more exposure to CPGs, screening, and stratification tools, and therefore be more likely to respond than clinicians who graduated earlier. Furthermore, the results might be skewed towards participants who use social media or other digital platforms through which the survey was predominantly advertised (Topolovec-Vranic \& Natarajan, 2016). Further research may benefit from more targeted recruitment methods. Finally, the results of this survey reflect participants' self-reported behaviours, which may not accurately represent their actual clinical practice; a situation which is in line with other similar studies conducted in New Zealand and internationally.

Analysis of the open-ended responses for specific questions indicated that some participants might have misunderstood some items. For example, some participants appeared confused by the definition of CPGs, and when asked which CPGs they used, responded with the McKenzie Mechanical Diagnosis and Therapy model and STarT Back, neither of which are CPGs. In these and similar instances, we did not change or correct any responses, but classified these answers as "other". These examples suggested that there is a general lack of understanding of CPGs, screening, and stratification tools, and supported the study's finding that further training and exposure is required to increase understanding, awareness, and clinical use of these tools.

\section{CONCLUSION}

This study was the first to investigate New Zealand physiotherapists' use of CPGs, screening, and stratification tools for LBP, as well as the perceived barriers to the use of the tools. Although just over half of the participants reported regularly using CPGs for LBP in clinical practice, the use of screening and stratification tools was much lower. Non-users of 
CPGs commonly noted that CPGs were unnecessary because they already knew how to treat people with LBP, they lacked training/exposure to CPGs, and the guidelines were out of date. In contrast, non-users of screening and stratification tools cited time constraints in clinical practice, lack of training/ exposure, and lack of resources as key barriers to using these tools. Significant associations were found between the use of screening tools and participants' level of qualification and years of experience, but no such associations were found for the use of CPGs or stratification tools. However, use of CPGs was significantly associated with use of stratification tools. Further research is required to better understand whether a stratified model of care for LBP may be implemented in New Zealand and whether this will improve outcomes, and additionally, what supports might be required to ensure implementation is successful.

\section{KEY POINTS}

1. Use of CPGs for LBP was reported by $53 \%$ of participants. Reasons for not using CPGs included participants relying on their clinical reasoning and knowledge to guide patient management, a lack of training/exposure, and the perception that CPGs needed to be updated with current evidence.

2. Although screening for psychosocial factors was nearly always incorporated into clinical practice, only $37 \%$ of participants regularly used screening tools, and there were inconsistencies in how these were used. Time constraints and lack of training/exposure were key barriers to their use.

3. Only $21 \%$ of participants reported using stratification tools in clinical practice, with lack of awareness, training, and time constraints identified as key barriers to their use.

4. Further research is required to better understand whether a stratified model of care for LBP may be implemented in New Zealand and what supports might be required to ensure implementation is successful.

\section{DISCLOSURES}

There are no conflicts of interest which may be perceived to interfere with or bias this study.

\section{PERMISSIONS}

Ethical approval was gained from the Auckland University of Technology Ethics Committee (reference number 19/72).

\section{ACKNOWLEDGEMENTS}

We would like to thank the following people: all participants for their time in completing the survey; Steve Ellery, Dr. Ramakrishnan Mani, and Kay Stevenson for reviewing the survey; and the Faculty of Health and Environmental Studies, Auckland University of Technology, and Keele University, United Kingdom, for providing funding for this research. We also acknowledge contributions made by the New Zealand STarT Back Group (a collaboration between the Auckland University of Technology and University of Otago).

\section{ADDRESS FOR CORRESPONDENCE}

Dr Julia Hill, Department of Physiotherapy, School of Clinical Sciences, Auckland University of Technology, Private Bag 92006, Auckland, New Zealand.

Email: julia.hill@aut.ac.nz

\section{REFERENCES}

Accident Compensation Corporation. (2004). New Zealand acute low back pain guide. https://www.healthnavigator.org.nz/media/1006/nz-acute-lowback-pain-guide-acc.pdf

Artus, M., van der Windt, D., Jordan, K. P., \& Croft, P. R. (2014). The clinical course of low back pain: A meta-analysis comparing outcomes in randomised clinical trials (RCTs) and observational studies. BMC Musculoskeletal Disorders, 15, 68. https://doi.org/10.1186/1471-2474-1568

Bernhardsson, S., Johansson, K., Öberg, B., Nilsen, P., \& Larsson, M. E. (2014). Determinants of guideline use in primary care physical therapy: A cross-sectional survey of attitudes, knowledge, and behavior. Physical Therapy, 94(3), 343-354. https://doi.org/10.2522/ptj.20130147

Childs, J. D., Fritz, J. M., Wu, S. S., Flynn, T. W., Wainner, R. S., Robertson, E. K., Kim, F. S., \& George, S. Z. (2015). Implications of early and guideline adherent physical therapy for low back pain on utilization and costs. BMC Health Services Research, 15, 150. https://doi.org/10.1186/s12913-0150830-3

Copeland, J. M., Taylor, W. J., \& Dean, S. G. (2008). Factors influencing the use of outcome measures for patients with low back pain: A survey of New Zealand physical therapists. Physical Therapy, 88(12), 1492-1505. https://doi.org/10.2522/ptj.20080083

Darlow, B., Fullen, B. M., Dean, S., Hurley, D. A., Baxter, G. D., \& Dowell, A. (2012). The association between health care professional attitudes and beliefs and the attitudes and beliefs, clinical management, and outcomes of patients with low back pain: A systematic review. European Journal of Pain, 16(1), 3-17. https://doi.org/10.1016/j.ejpain.2011.06.006

Dunn, K. M., Hestbaek, L., \& Cassidy, J. D. (2013). Low back pain across the life course. Best Practice \& Research Clinical Rheumatology, 27(5), 591-600. https://doi.org/10.1016/j.berh.2013.09.007

Dykema, J., Jones, N. R., Piché, T., \& Stevenson, J. (2013). Surveying clinicians by web: Current issues in design and administration. Evaluation and the Health Professions, 36(3), 352-381. https://doi. org/10.1177/0163278713496630

Foster, N. E., \& Delitto, A. (2011). Embedding psychosocial perspectives within clinical management of low back pain: Integration of psychosocially informed management principles into physical therapist practice challenges and opportunities. Physical Therapy, 91(5), 790-803. https:// doi.org/10.2522/ptj.20100326

Foster, N. E., Hill, J. C., O'Sullivan, P., \& Hancock, M. (2013). Stratified models of care. Best Practice \& Research Clinical Rheumatology, 27, 649-661. https://doi.org/10.1016/j.berh.2013.10.005

Foster, N. E., Mullis, R., Hill, J. C., Lewis, M., Whitehurst, D. G., Doyle, C., Konstantinou, K., Main, C., Somerville, S., Sowden, G., Wathall, S., Young, J., \& Hay, E. M; IMPaCT Back Study team. (2014). Effect of stratified care for low back pain in family practice (IMPaCT back): A prospective population-based sequential comparison. Annals of Family Medicine, 12(2), 102-111. https://doi.org/10.1370/afm.1625

Green, D. J., Lewis, M., Mansell, G., Artus, M., Dziedzic, K. S., Hay, E. M., Foster, N. E., \& van der Windt, D. A. (2018). Clinical course and prognostic factors across different musculoskeletal pain sites: A secondary analysis of individual patient data from randomised clinical trials. European Journal of Pain, 22(6), 1057-1070. https://doi.org/10.1002/ejp.1190

Hendrick, P., Mani, R., Milosavljevic, S., Schneiders, A. G., \& Bishop, A. (2013). Therapist knowledge, adherence and use of low back pain guidelines to inform clinical decisions - A national survey of manipulative and sports physiotherapists in New Zealand. Manual Therapy, 18(2), 136142. https://doi.org/10.1016/j.math.2012.09.002 
Hill, J. C., Vohora, K., Dunn, K. M., Main, C. J., \& Hay, E. M. (2010). Comparing the STarT back screening tool's subgroup allocation of individual patients with that of independent clinical experts. Clinical Journal of Pain, 26(9), 783-787. https://doi.org/10.1097/ AJP.0b013e3181f18aac

Hill, J. C., Whitehurst, D. G., Lewis, M., Bryan, S., Dunn, K. M., Foster, N. E., Konstantinou, K., Main, C. J., Mason, E., Somerville, S., Sowden, G., Vohora, K., \& Hay, E. M. (2011). Comparison of stratified primary care management for low back pain with current best practice (STarT Back): A randomised controlled trial. The Lancet, 378(9802), 1560-1571. https:// doi.org/10.1016/S0140-6736(11)60937-9

Hingorani, A. D., van der Windt, D. A., Riley, R. D., Abrams, K., Moons, K. G., Steyerberg, E. W., Schroter, S., Sauerbrei, W., Altman, D. G., \& Hemingway, H; PROGRESS Group. (2013). Prognosis research strategy (PROGRESS) 4: Stratified medicine research. BMJ, 346:e5793. https://doi. org/10.1136/bmj.e5793

Hodges, P. W. (2019). Hybrid approach to treatment tailoring for low back pain: A proposed model of care. Journal of Orthopaedic \& Sports Physical Therapy, 49(6), 453-463. https://doi.org/10.2519/ jospt.2019.8774

Itz, C. J., Geurts, J. W., van Kleef, M., \& Nelemans, P. (2013). Clinical course of non-specific low back pain: A systematic review of prospective cohort studies set in primary care. European Journal of Pain, 17(1), 5-15. https:// doi.org/10.1002/j.1532-2149.2012.00170.x

Karstens, S., Wensing, M., Krug, K., Szecsenyi, J., Kuithan, P., Joos, S., Hill, J. C., \& Steinhäuser, J. (2018). Physiotherapists' views of implementing a stratified treatment approach for patients with low back pain in Germany: A qualitative study. BMC Health Services Research, 18(1), 214. https://doi. org/10.1186/s12913-018-2991-3

Khazaal, Y., van Singer, M., Chatton, A., Achab, S., Zullino, D., Rothen, S., Khan, R., Billieux, J., \& Thorens, G. (2014). Does self-selection affect samples' representativeness in online surveys? An investigation in online video game research. Journal of Medical Internet Research, 16(7), e164. https://doi.org/10.2196/jmir.2759

Lin, I., Wiles, L., Waller, R., Goucke, R., Nagree, Y., Gibberd, M., Straker, L., Maher, C. G., \& O'Sullivan, P. (2019). What does best practice care for musculoskeletal pain look like? Eleven consistent recommendations from high-quality clinical practice guidelines: Systematic review. British Journal of Sports Medicine, 54(2), 79-86. https://doi.org/10.1136/ bjsports-2018-099878

Menezes Costa, L. D. C., Maher, C. G., Herbert, R. D., Hancock, M. J., McAuley, J. H., \& Costa, L. O. P. (2012). The prognosis of acute and persistent low-back pain: A meta-analysis. Canadian Medical Association Journal, 184(11), 613-624. https://doi.org/10.1503/ cmaj. 111271

Miki, T., Kondo, Y., Takebayashi, T., \& Takasaki, H. (2020). Difference between physical therapist estimation and psychological patient-reported outcome measures in patients with low back pain. PLoS One, 15(1), e0227999. https://doi.org/10.1371/journal.pone.0227999

Ministry of Health. (2016). Health loss in New Zealand 1990-2013: A report from the New Zealand burden of diseases, injuries and risk factors study. https://www.health.govt.nz/system/files/documents/publications/healthloss-in-new-zealand-1990-2013-aug16.pdf
National Health Committee. (2015). Low back pain: A pathway to prioritisation. https://www.health.govt.nz/system/files/documents/ publications/nhc-lbp-pathway-to-prioritisation.pdf

National Institute for Health and Care Excellence. (2016). Low back pain and sciatica in over 16s: Assessment and management. NICE guideline [NG59]. https://www.nice.org.uk/guidance/ng59

O’Sullivan, P., Caneiro, J. P., O’Keeffe, M., \& O'Sullivan, K. (2016). Unraveling the complexity of low back pain. Journal of Orthopaedic \& Sports Physical Therapy, 46(11), 932-937. https://doi.org/10.2519/ jospt.2016.0609

Oliveira, C. B., Maher, C. G., Pinto, R. Z., Traeger, A. C., Lin, C-W. C., Chenot, J. F., van Tulder, M., \& Koes, B. W. (2018). Clinical practice guidelines for the management of non-specific low back pain in primary care: An updated overview. European Spine Journal, 27(11), 2791-2803. https:// doi.org/10.1007/s00586-018-5673-2

Physiotherapy Board of New Zealand. (2019). Annual report 2019. https:// www.physioboard.org.nz/wp-content/uploads/2019/09/V6-Physio-AnnualReport-2019.pdf

Ramond, A., Bouton, C., Richard, I., Roquelaure, Y., Baufreton, C., Legrand, E., \& Huez, J. F. (2011). Psychosocial risk factors for chronic low back pain in primary care - A systematic review. Family Practice, 28(1), 12-21. https://doi.org/10.1093/fampra/cmq072

Sowden, G., Hill, J. C., Morso, L., Louw, Q., \& Foster, N. E. (2018). Advancing practice for back pain through stratified care (STarT Back). Brazilian Journal of Physical Therapy, 22(4), 255-264. https://doi.org/10.1016/j. bjpt.2018.06.003

Steyerberg, E. W., Moons, K. G., van der Windt, D. A., Hayden, J. A., Perel, P., Schroter, S., Riley, R. D., Hemingway, H., \& Altman, D. G; PROGRESS Group. (2013). Prognosis research strategy (PROGRESS) 3: Prognostic model research. PLoS Medicine, 10(2), e1001381. https://doi.org/10.1371/ journal.pmed.1001381

Synnott, A., O'Keeffe, M., Bunzli, S., Dankaerts, W., O'Sullivan, P., \& O'Sullivan, K. (2015). Physiotherapists may stigmatise or feel unprepared to treat people with low back pain and psychosocial factors that influence recovery: A systematic review. Journal of Physiotherapy, 61(2), 68-76. https://doi.org/10.1016/j.jphys.2015.02.016

Topolovec-Vranic, J., \& Natarajan, K. (2016). The use of social media in recruitment for medical research studies: A scoping review. Journal of Medical Internet Research, 18(11), e286. https://doi.org/10.2196/ jmir.5698

Tumilty, S., Adhia, D. B., Rhodes, R., \& Mani, R. (2017). Physiotherapists treatment techniques in New Zealand for management of acute nonspecific low back pain and its relationships with treatment outcomes: A pilot study. Physical Therapy Reviews, 22(1-2), 95-100. https://doi.org/1 0.1080/10833196.2017.1282073

Zusman, M. (2013). Belief reinforcement: One reason why costs for low back pain have not decreased. Journal of Multidisciplinary Healthcare, 6, 197-204. https://doi.org/10.2147/JMDH.S44117 